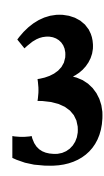

\title{
TENDENCIAS EN LA EVALUACIÓN DEL APRENDIZAJE EN CURSOS EN LÍNEA MASIVOS Y ABIERTOS
}

\author{
(TRENDS IN ASSESSMENT IN MASSIVE OPEN ONLINE COURSES)
}

María Jesús Gallego-Arrufat

Vanesa Gámiz-Sánchez

Universidad de Granada

Elba Gutiérrez-Santiuste

Universidad de Córdoba

DOI: 10.5944/educXX1.12935

Cómo referenciar este artículo/How to reference this article:

Gallego-Arrufat, M. J.; Gámiz-Sánchez, V. y Gutiérrez-Santiuste, E. (2015). Tendencias en la evaluación del aprendizaje en cursos en línea masivos y abiertos. Educación XX1, 18(2), 77-96, doi: 10.5944/educXX1.12935

Gallego-Arrufat, M. J.; Gámiz-Sánchez, V. \& Gutiérrez-Santiuste, E. (2015). Trends in assessment in massive open online courses. Educación XX1, 18(2), 77-96, doi: 10.5944/educXX1.12935

\section{RESUMEN}

La evaluación del aprendizaje es una característica central en el diseño pedagógico de cursos en línea masivos y abiertos (MOOC) (Sandeen, 2013). Aunque el fenómeno MOOC es nuevo, la literatura sobre evaluación electrónica (e-assessment) proporciona una base de conocimiento derivado de la investigación hacia el necesario apoyo del aprendizaje de los participantes. Unida a la evaluación, la satisfacción de los participantes en los MOOC adquiere un protagonismo especial, teniendo en cuenta el interés que suscitan los cursos y las elevadas tasas de abandono que sufren. El objetivo de este artículo es doble, por una parte el análisis de los procesos de evaluación que se desarrollan en 87 cursos de diferentes plataformas de MOOC (internacionales, de universidades españolas y otras) y, por otra, la reflexión sobre esos procesos basada en el estudio de MOOC desarrollados en la Universidad de Granada (España). Se describen los resultados agrupados en: qué se evalúa, quién realiza la evaluación, momento en que se efectúa, instrumentos empleados, tipo de evaluación y carácter de la certificación (participación/acreditación). Se estudia la satisfacción sobre la evaluación de 516 participantes de la primera edición de MOOC ofertados por AbiertaUGR en 2013. La satisfacción de los participantes está relacionada con la interactividad y el aprendizaje percibido se corres- 
ponde con una evaluación formativa o evaluación para el aprendizaje. Se aportan propuestas de mejora sobre el diseño pedagógico de la evaluación en futuros MOOC.

\section{PALABRAS CLAVE}

E-evaluación; e-learning; cursos en línea; MOOC; satisfacción; tecnología educativa.

\section{ABSTRACT}

Assessment is a central characteristic in the design of massive open online courses (MOOCs) (Sandeen, 2013). Although this phenomenon is new, the literature on e-assessment suggests a direction based on a knowledge base derived from research into the support needs of the participants' learning. Together with assessment, participant satisfaction with MOOCs takes on a special role when we consider the interest of the courses and their high drop-out rate. We have two objectives in this paper; on one hand, we analyze the assessment processes in 87 MOOCs developed on different international platforms (Coursera, Udacity, MiríadaX, EdX, and RedunX) at Spanish universities (AbiertaUGR, UPVX, UniMOOC, UnedComa, and Ehusfera) and others; while on the other hand, we reflect on these processes assisted by the study of the MOOCs developed at the University of Granada (Spain). We describe the results classified according to: what is assessed, who performs the assessment, time at which assessment occurs, instruments employed, type of assessment, and type of the certification (participation/accreditation). We study the satisfaction of 513 participants in courses of the first edition offered by AbiertaUGR in 2013. Participant satisfaction is related to interactivity, and the learning perceived corresponds to a formative assessment or assessment for learning. Suggestions for improvement are given for the pedagogical design of the assessment in future MOOCs.

\section{KEY WORDS}

E-assessment; e-learning; online courses; MOOC; satisfaction; educational technology.

\section{INTRODUCCIÓN}

La evaluación de aprendizajes constituye uno de los pilares esenciales en los procesos de diseño y desarrollo en los cursos en línea, con un énfasis especial en la evaluación formativa o evaluación para el aprendizaje (Lawton, Vye et al., 2012; Vonderwell \& Boboc, 2013). También en el caso de 
los cursos en línea masivos y abiertos (massive open online courses, MOOC), fenómeno iniciado por George Siemens en la Universidad de Athabasca y Stephen Downes en el National Research Council de Canadá.

Algunos MOOC están basados en el conectivismo y el networking, dentro de un ambiente abierto. Otros son más behavioristas, con tareas y objetivos de aprendizaje específicos. En cualquier caso tienen un patrón que atrae a gran número de participantes (ej. 155.000 en un MOOC del MIT) con una tasa de deserción muy alta, 90\%-87\% (Agarwala, 2013; Jordan, 2014), si bien en algunos casos es menor (King, Robinson \& Vickers, 2014). Se critica que algunos descansan en la actualidad o moda del tema y/o en un académico ampliamente conocido más que en el diseño pedagógico de un curso válido. Probablemente sea debido a la falta de diseños de instrucción eficaces y a la escasez de investigación rigurosa sobre el tema.

Dentro del universo MOOC, la evaluación es una característica central del diseño desde el principio. En este nuevo contexto, la evaluación se dirige menos al cumplimiento o rendimiento de cuentas y más al apoyo del aprendizaje de los participantes. En última instancia, el éxito del estudiante y el logro se sitúan en una posición central, como debe ser (Sandeen, 2013).

El diseño pedagógico debería ser clave en las actividades formativas propias de un MOOC, relacionándose la evaluación electrónica en el mismo (e-assessment) con la necesaria interactividad del curso. Si se pretende un aprendizaje autorregulado, es preciso estudiar el grado de satisfacción experimentado por los participantes en los cursos, y de ahí derivar pautas para el diseño de una e-evaluación apropiada en MOOC. Estos son los ejes principales de la revisión del estado de la cuestión en esta investigación: la e-evaluación en el diseño pedagógico de MOOC y la satisfacción de los participantes ante la e-evaluación en MOOC.

\section{E-EVALUACIÓN EN EL DISEÑo PEDAGógICo DE MOOC}

Indudablemente, para que la evaluación sea efectiva, debe ser parte de una teoría pedagógica. La e-evaluación debe ser integrada en una visión holística de la pedagogía. Esto significa que cualquier teoría de la evaluación presupone e informa a una teoría del aprendizaje. Se propone diferenciar entre conectivismo en MOOC (cMOOCs) y los xMOOCs (Siemens, 2012; Baggaley, 2013). Siemens y Downes, pioneros del conectivismo en MOOC (cMOOC) argumentaron que ni behaviorismo, cognitivismo o constructivismo explican adecuadamente lo que ocurre en la Web 2.0 (Clarà y Barberà, 2013). 
Se ha demostrado que la evaluación se encuentra en la base de las diferencias entre la construcción del conocimiento y el aprendizaje e influye en las discusiones escritas de participantes en entornos online (Cheng, Jordan \& Schallert, 2013). Los hallazgos sugieren que el modo en que los participantes interactúan en línea está influido por la forma de evaluación de sus discusiones. También que el conocimiento y el aprendizaje están relacionados pero son constructos distintos, correlacionados dentro de cada contexto de escritura, disociados en distintos contextos, y que actúan de manera diferencial según la percepción de las demandas académicas. En estos nuevos cursos en línea la evaluación del aprendizaje tradicional se encuentra limitada.

De ahí la necesidad de estudiar la evaluación electrónica (e-assessment) en entornos donde predomina el carácter abierto y la necesidad de encontrar información sobre su adecuación en esta nueva modalidad de MOOC. Aunque la evidencia empírica directamente relacionada con los MOOC en la actualidad aún es difícil de encontrar y la discusión de los modelos de evaluación de aprendizaje en MOOC no está muy desarrollada (Haggard, 2013), se sugiere que no hay ninguna razón para creer que sean menos eficaces que una experiencia de aprendizaje de compañeros face-to-face (Glance, Forsey \& Riley, 2013).

En todo caso, se estima que la evaluación electrónica en cursos abiertos posee características especiales, añadidas a las de certificación y acreditación propias de los MOOC debido a su carácter abierto. Y sería importante evitar ciertos errores cometidos en ocasiones en el diseño de la evaluación del aprendizaje en cursos en línea (Shank, 2012), tales como esperar una curva de aprendizaje con forma de campana, elegir un tipo incorrecto de evaluación, evaluaciones insuficientes o tests de elección múltiple mal redactados.

En el contexto español la investigación sobre e-evaluación es llevada a cabo en estudios en pequeña escala (Yuste, Alonso y Blázquez, 2012; Lafuente, Remesal y Álvarez, 2014) predominando cuestiones cerradas y de elección múltiple (Ramsaran-Fowdar, Baguant \& Fowdar, 2011; Stödberg, 2012; Al-Smadi \& Gueti, 2011). En este tipo de cuestiones las respuestas son simples, por lo que en algunos estudios se demanda, con apoyo educativo explícito mediante las TIC, negociar significados con los participantes e incrementar su autonomía y aprendizaje autorregulado (Cebrián, 2012; Lafuente, Remesal y Álvarez, 2014). También se ha demostrado que un diseño metodológico que haga hincapié en los conceptos esenciales y un feedback formativo pueden producir diferencias significativas en los resultados de aprendizaje en cursos en línea (Lawton, Vye et al., 2012).

Para llegar a una evaluación formativa, merece especial atención la necesaria interactividad, teniendo en cuenta los intercambios comunicacionales 
anteriores y posteriores, extensivos e intensivos, que se producen alrededor de la evaluación para el aprendizaje. Se han propuesto instrumentos como la e-rúbrica (Cebrián, 2012), modelos para integrar objetos de aprendizaje interactivos (Saul \& Wuttke, 2012); e intentos de evaluar preguntas abiertas mediante la interacción y la colaboración de los participantes (Hoang \& ArchInt, 2013).

Los principios de la evaluación no cambian en un entorno electrónico. La e-evaluación se sustenta en los mismos principios de validez, flexibilidad y equidad y también utiliza las mismas estrategias que los métodos de evaluación tradicionales (Brink \& Lautenbach, 2011), aunque probablemente sea preciso contar con mayores niveles de interactividad en cursos en línea para favorecer tanto el seguimiento del progreso como la motivación del estudiante.

Como en cualquier otra modalidad de evaluación del aprendizaje (Lukas y Santiago, 2004; De la Herrán, 2014) en su análisis es preciso distinguir qué, quién, cuándo y cómo se evalúa (que comprende tanto el tipo de evaluación -continua, formativa, de proceso, normativa-, como los distintos instrumentos de evaluación empleados). En el caso de los MOOC cabe añadir el para qué, es decir, si es requisito o no la evaluación para la obtención de certificación y acreditación en el curso (Anderson \& McGreal, 2012).

\section{SATISFACCIÓN ANTE LA E-EVALUACIÓN DE LOS PARTICIPANTES EN MOOC}

Existe un interés creciente de los investigadores por averiguar las visiones de los estudiantes sobre el modo en que la tecnología les ayuda a satisfacer las crecientes demandas en un entorno cambiante. En este marco se atiende a la evaluación, en mayor medida en Educación Superior y en entornos de aprendizaje en línea (Earl, 2013).

También existen investigaciones sobre el punto de vista de los participantes en MOOC (Zutshi, O'Hare \& Rodafinos, 2013) y sus niveles de satisfacción, que llegan en alguna ocasión al 91\% (Kerrison, 2013), si bien son más escasas aquellas que se centran en aspectos concretos, como la evaluación. Sun, Tsai et al. (2008) han encontrado que la satisfacción percibida por el participante está influida por la flexibilidad y calidad de los cursos en línea, la utilidad percibida y la facilidad de uso, así como la existencia de tipos diversos de evaluación. Mackness, Mak \& Willians (2010) relacionan la satisfacción con la interactividad, partiendo de la idea de Siemens de que la cuestión no es el modo en que los participantes se conectan sino las implicaciones de que la gente se conecte de una forma determinada. La conectividad no asegura 
necesariamente la interacción, siendo necesario superar barreras para mejorar no solo la conectividad sino también la interacción. Los participantes expresan diferentes niveles de satisfacción en función, no tanto de la cantidad, sino de la calidad de las interacciones. En la base se encuentra la presencia cognitiva, presencia docente y presencia social características del modelo de CoI («Community of Inquiry») (Gallego-Arrufat y Gutiérrez-Santiuste, 2015). El aumento de la interacción eleva el nivel de satisfacción.

En la investigación de Sorensen (2013) se encuentra un alto grado de participación y compromiso en los participantes y buenos resultados. La encuesta en línea tras el curso aporta el resultado de que son los de mayor rendimiento quienes se encuentran más satisfechos con este sistema. Para los participantes, la e-evaluación tiene retos (como la honestidad académica) y beneficios (como la posibilidad de obtener feedback inmediato). Por su parte, la disponibilidad de instrumentos y tecnologías proporciona una variedad de tipos de evaluación para los diseñadores (Meyer \& Zhu, 2013).

En esta investigación el propósito es doble: por un lado describir y comprender los procesos de evaluación propuestos en MOOC para obtener datos sobre las tendencias de las actividades evaluativas propias de estos cursos; y por otro - en relación con lo anterior - realizar un estudio intensivo de la satisfacción que experimentan los participantes en MOOC de la plataforma AbiertaUGR sobre la e-evaluación.

\section{MATERIAL Y MÉTODOS}

El objetivo fundamental de este trabajo es el análisis de los procesos de evaluación que se desarrollan en MOOC y la reflexión sobre esos procesos basada en un estudio de caso llevado a cabo en la Universidad de Granada (España). Se establecen para ello dos fases diferenciadas: una primera fase consistente en un análisis exploratorio de las características de la evaluación en una colección de MOOC y una segunda fase en la que se realiza el estudio de un caso centrado en las percepciones manifestadas por sus participantes.

\section{Fase 1. Análisis de la evaluación empleada en MOOC}

La muestra inicial es de 129 MOOC sometida a análisis por pedagogos en formación en un curso universitario sobre tecnologías en educación. Los MOOC fueron seleccionados a través de un muestreo incidental con un posible sesgo debido a los algoritmos que utiliza el motor de búsqueda Google y los sistemas de recomendación. Se les solicitó una descripción escrita del sistema de evaluación de los MOOC que habían elegido libremente. El equipo investigador efectuó un filtrado donde se validaron aquellos re- 
gistros con las características de un curso MOOC (abierto, online y gratuito), con información sobre su evaluación y de plataformas reconocidas (Scopeo, 2013). La muestra final consta de 87 MOOC.

La distribución de los cursos según plataformas de MOOC representa las principales internacionales y nacionales (Oliver, Hernández-Leo y otros, 2014):

- Coursera y MiríadaX tienen una representación en el estudio superior al 25\% de los MOOC analizados, cada una de ellas.

- Unedcoma, AbiertaUGR y UPVX representan entre el $5 \%$ y el 15\% de los MOOC analizados, cada una de ellas.

- Inferior al 5\% de los MOOC analizados pertenecen a cada una de las siguientes: Udacity, Interfmooc, UniMOOC-AEmprendemooc, Acamica, Guadalinfomooc, Ehusfera, Open2Study, RedunX, TelecomBretagne, Telescopio Galileo, Unimooc, EdX.

Las temáticas de los MOOC analizados son diversas: Ciencias Sociales (41.07\%), Humanidades (17.64\%), Ciencias Naturales (0.58\%), Ciencias de la Salud $(9.8 \%)$, Tecnología $(17.64 \%)$ y otros contenidos $(13.72 \%)$.

Se ha construido una base de datos en FileMaker Pro v.1 para realizar un análisis de contenido de las descripciones escritas de la evaluación en los MOOC. Los datos categorizados han sido obtenidos a través de un proceso inductivo. Los campos en cada uno de los registros son: identificador, plataforma, y cuatro veces repetidos los campos: qué se evalúa (objeto de evaluación), quién realiza la evaluación (agente que la realiza), cuándo (momento en que se realiza la evaluación), herramienta de evaluación (instrumento para realizarla), tipo de evaluación, requisito para diploma de participación (si es un requerimiento para la obtención del diploma de participación), requisito para diploma de superación (si es un requerimiento para la obtención del diploma de superación), porcentaje de valoración en la evaluación final y otros. Esta repetición de campos se ha realizado hasta alcanzar el número máximo de herramientas de evaluación encontradas, registrándose hasta cuatro herramientas de evaluación en un mismo curso. Se ha procedido a la revisión de la categorización realizada para encontrar posibles sesgos por dos miembros del equipo investigador, habiendo sido corregidas las discordancias.

\section{Fase 2. Estudio de caso}

El caso seleccionado para profundizar en la evaluación en los MOOC, uno de los analizados en la fase anterior, es el ciclo de cursos organizados en 2013 por el Centro de Enseñanzas Virtuales de la Universidad de Granada 
en su iniciativa AbiertaUGR (Gea y Montes, 2013) titulado «Tecnologías Digitales, Internet y Aprendizaje 2.0». Consta de tres cursos MOOC piloto de cuatro semanas de duración cada uno. El primer curso, «Identidades Digitales», sobre la comunicación, la interacción y la actividad social en Internet para gestionar la propia identidad digital. El segundo, «Aprendizaje ubicuo", sobre aprendizaje basado en TIC y en dispositivos móviles/tablets. El tercero, "Licencias Creative Commons y recursos abiertos en Internet», centrado en cómo publicar en Internet materiales en abierto. El número de participantes inscritos es 1771, 992 y 3046, respectivamente, y para su difusión solo se utilizan las redes sociales y su propia página web.

El sistema de evaluación en los cursos es de valoración automática de los logros por medio de puntos, que se pueden conseguir por: acceso a la plataforma (controlando el tiempo entre accesos), edición del perfil e información de usuario, comentarios en discusiones y debates o uso de blogs. Para ello, cada semana se proponen actividades para desarrollar dentro de la plataforma (a través de debates, discusiones, blogs) o fuera (con comentarios dentro de ella). El diseño contempla que un usuario medio puede conseguir entre 15 y 25 puntos semanales. Un sistema de reconocimiento automático otorga insignias o medallas (badges) cada vez que el usuario supera ese nivel (McDaniel, Lindgren \& Friskics (2012). Para superar el curso hay que alcanzar como mínimo el tercero de un total de cinco badges, que representa el $50 \%$ de la puntuación máxima estimada para el curso (normativa, se compara con el resto). Además semanalmente se otorgan otras tres medallas para destacar a determinados participantes por su actividad en el curso: Sociable, Participativo y Emprendedor. El tipo de evaluación realizada es procesual y continua, ya que se desarrolla a lo largo de todo el proceso, y normativa, ya que los criterios se establecen en relación con el nivel medio planificado para el grupo.

Para el análisis de resultados, se recoge la opinión de los usuarios manifestada a través de una encuesta de satisfacción al terminar el curso. El cuestionario, de carácter voluntario, es completado por 241 participantes del primer curso (13,61\% de los inscritos), 185 del segundo $(18.64 \%)$ y 90 del tercero $(2,95 \%)$. Además de cuestiones de carácter general, como una valoración global del curso, realizan una pregunta de respuesta abierta sobre los aspectos que mejorarían según su experiencia. La información es recogida y catalogada en unidades temáticas de las que se extraen las referidas explícitamente a la evaluación. Posteriormente, sobre esta selección se procede a la categorización de los datos para la realización de un análisis de contenido a través de un proceso inductivo. Las categorías obtenidas, validadas por varios miembros del equipo investigador, son: opinión positiva (respuestas que elogian la evaluación), necesidad de feedback (aumento de unidades de retroalimentación dentro del curso), comentarios inadecuados (intervenciones 
en los foros), sistema de evaluación (en conjunto), más instrumentos, mayor información sobre el sistema de evaluación, mayor interoperabilidad, mayor personalización.

\section{RESULTADOS}

\section{Estrategias de evaluación empleadas en MOOC}

De los 87 MOOC analizados se ha extraído la información necesaria para dar respuesta al primer objetivo de investigación. El análisis de la evaluación del aprendizaje en los MOOC se ha estructurado en dos secciones: por un lado, determinar el qué, quién, cuándo, cómo y para qué de la evaluación en la muestra analizada y, por otro, realizar un análisis de las herramientas según las plataformas de los cursos.

La Figura 1 muestra los resultados de ¿qué se evalúa en los MOOC? Aparecen en primer lugar conocimientos y contenidos, seguida de la satisfacción del participante con el curso (evaluada también en el 16.13\% de los MOOC analizados). Se observa la tendencia señalada por Aguaded, Vázquez-Cano \& Sevillano-García (2013) con predominio de xMOOC orientados a contenidos más que cMOOC basados en colaboración o interacción.

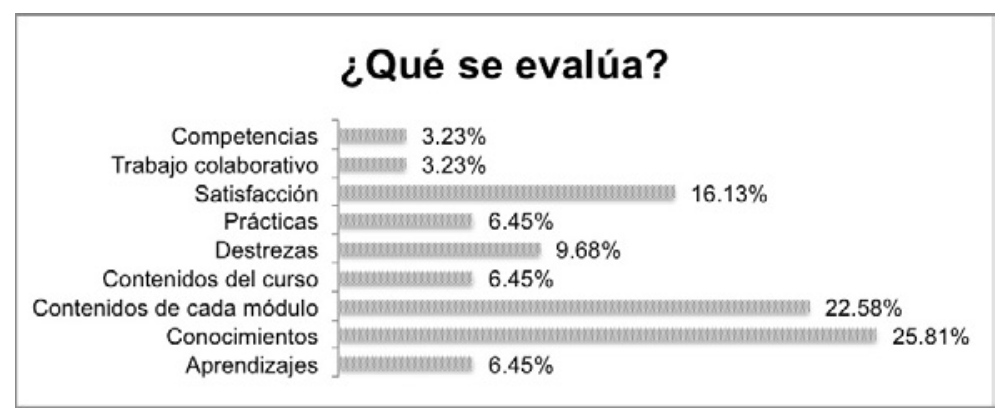

Figura 1. Qué se evalúa

Según quién efectúa la evaluación, más de la mitad de los cursos analizados contemplan una evaluación entre pares que, como señalan Glance, Forsey \& Riley (2013), es característica en la ejecución de los cursos masivos, proporcionando una información rápida sobre los aprendizajes. En el 10.17\% de los cursos analizados se ha hallado una coevaluación entre profesorado y alumnado. También aparece la evaluación automatizada que aporta una retroalimentación instantánea de los errores y aciertos obtenidos e incrementa la interactividad. 


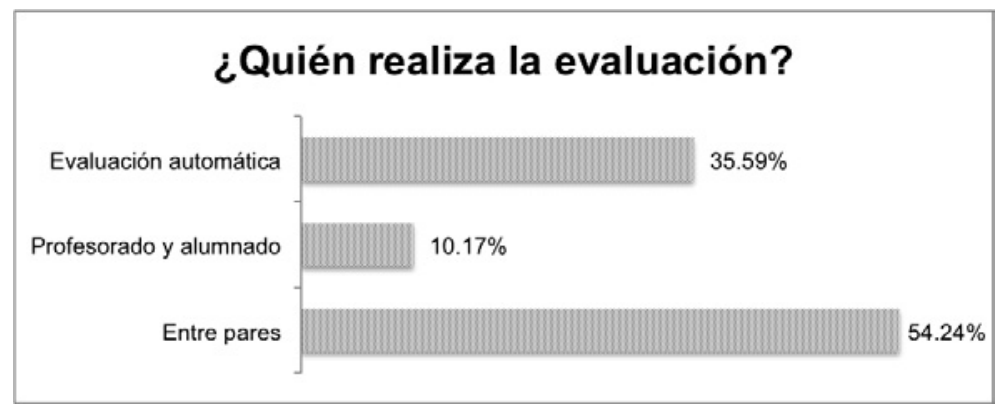

Figura 2. ¿Quién evalúa?

El periodo temporal de la evaluación es variable en los MOOC (Figura 3). Se observa que coexisten varios momentos de evaluación, realizándose una distribución a lo largo del desarrollo del curso junto con una evaluación final (35\%).

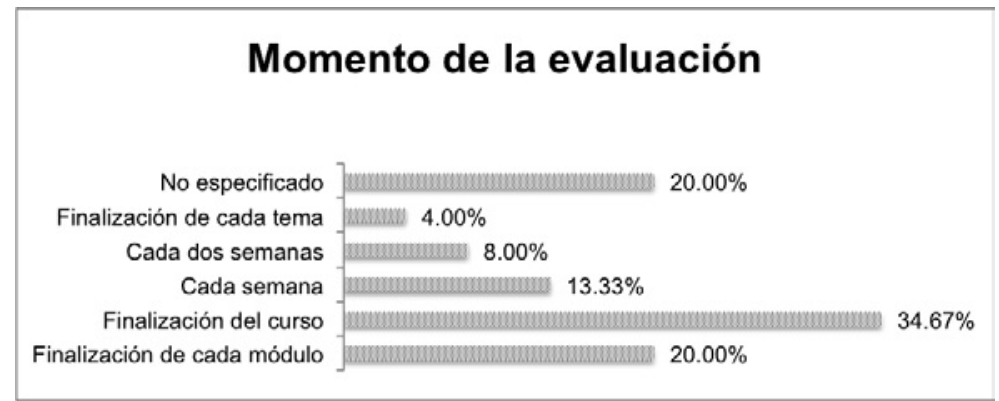

Figura 3. Momento de la evaluación

En la Figura 4 aparecen las herramientas e instrumentos utilizados para la evaluación del aprendizaje en MOOC. La categoría actividades incluye tareas o ejercicios diversos y acordes con la temática de cada curso. Los cuestionarios oscilan entre 8 y 32 ítems, son de tipo test con 4 o 5 respuestas, corrección automatizada y autoevaluación, unos sin posibilidad de repetición, otros con un número máximo de intentos y otros ofrecen la posibilidad de ser repetidos sin límite. Se observa que la categoría rúbrica es muy poco utilizada. Los trabajos contemplan tanto individuales como en equipos. Los accesos están referidos al número de veces que accede el participante al curso y las medallas sociales a las otorgadas por los compañeros. 


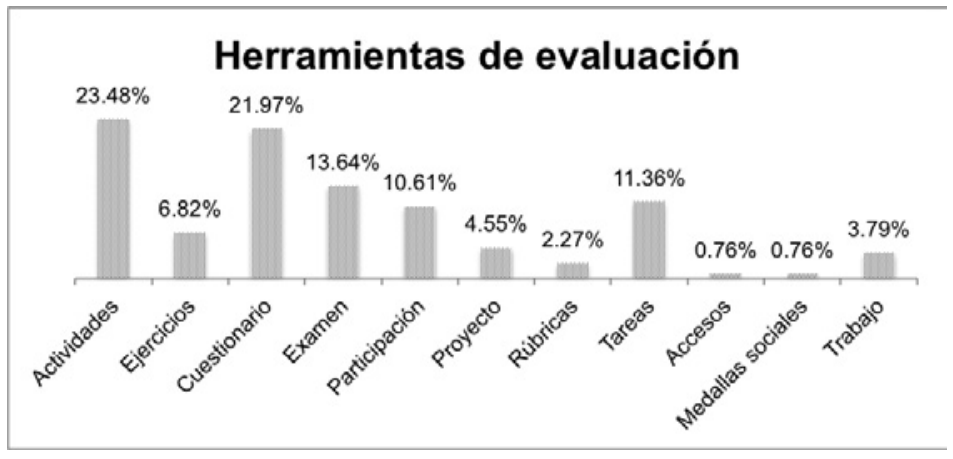

Figura 4. Herramientas de evaluación

Descartando en la investigación aquellos MOOC que no señalan, al menos, una herramienta de evaluación, los datos muestran una relación decreciente entre el número de evaluaciones realizadas y el porcentaje de MOOC que las contempla (Figura 5).

\section{Número \\ de herramientas de evaluación}

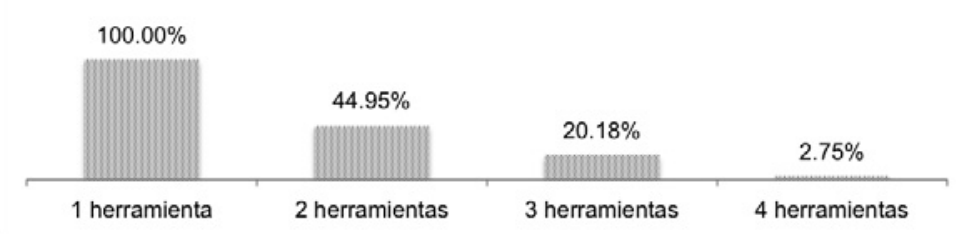

Figura 5. Número de herramientas de evaluación en cada MOOC

Son variadas las tipologías de evaluación en los MOOC analizados (Figura 6). Según la temporalización se produce una evaluación procesual y continua que aporta información significativa de los avances del aprendizaje (Figura 3). También se han hallado MOOC cuya orientación evaluativa es eminentemente normativa tomando como referente el rendimiento del grupo y su normalidad, incluyendo la excepcionalidad tanto inferior como superior (De la Herrán, 2014). En relación a la finalidad de la evaluación el $14,29 \%$ señalan que es formativa entendida como evaluación por pares o autoevaluación. 


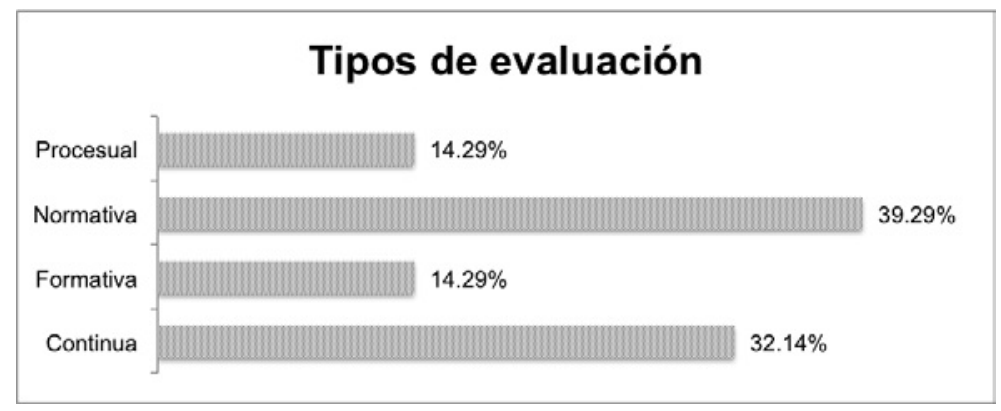

Figura 6. Tipos de evaluación

El $3.1 \%$ de las herramientas evaluativas analizadas son un requisito para avanzar hacia el siguiente módulo. El $0.6 \%$ señala expresamente que la evaluación es requisito para la certificación de participación y el $0.5 \%$ señala expresamente que la evaluación es requisito para la certificación de superación. En uno de los MOOC analizados se contempla una puntuación mínima para la obtención de ambas certificaciones.

La Tabla 1 refleja el análisis de las herramientas de evaluación según las plataformas MOOC. Estos hallazgos están referidos a los MOOC analizados y no supone que las plataformas tengan únicamente los señalados.

Tabla 1

Herramientas para la evaluación del aprendizaje en diferentes plataformas

\begin{tabular}{|c|c|c|c|c|c|c|c|c|c|}
\hline & Actividades & Ejercicios & Cuestionario & Examen & Participación & Proyecto & Rúbricas & Tareas & Trabajo \\
\hline \multicolumn{10}{|c|}{ Gestionadas directamente por universidades } \\
\hline Abierta UGR & & & & & & & & & $x$ \\
\hline UPVX & $x$ & & $\mathrm{x}$ & $\mathrm{x}$ & & & & & \\
\hline Ehusfera & $x$ & & $x$ & & & & & & \\
\hline Unedcoma & $x$ & & $x$ & $x$ & $x$ & & & $x$ & $x$ \\
\hline Coursera & $x$ & $x$ & $x$ & $x$ & $x$ & $x$ & $x$ & $x$ & \\
\hline Open2Study & $x$ & & $\mathrm{x}$ & & & & & & \\
\hline $\begin{array}{l}\text { Telescopio } \\
\text { Galileo }\end{array}$ & $\mathrm{x}$ & & & & & & & & \\
\hline \multicolumn{10}{|c|}{ Gestionadas por otras instituciones públicas o privadas } \\
\hline Acamica & & & & $\mathrm{x}$ & & & & & \\
\hline Guadalinfomo $\propto \mathrm{C}$ & & & $x$ & & & & & & \\
\hline $\begin{array}{l}\text { EducaLab- } \\
\text { Interfmooc }\end{array}$ & $x$ & & & & & & & & \\
\hline MiriadaX & $x$ & & $x$ & $x$ & & & & $x$ & $x$ \\
\hline Redunx & $x$ & & & & & & & & \\
\hline $\begin{array}{l}\text { Telecom- } \\
\text { bretagne }\end{array}$ & & & $\mathrm{x}$ & & & & & & \\
\hline Udacity & & & & $x$ & & $x$ & & & \\
\hline $\begin{array}{l}\text { Unimooc- } \\
\text { AEmprende }\end{array}$ & & & $x$ & & & & & & \\
\hline $\mathrm{EdX}$ & $x$ & & $x$ & & & & & & \\
\hline
\end{tabular}

El 16.13\% de los MOOC analizados realizan encuestas de satisfacción (Figura 1) como herramienta de evaluación. Una de las plataformas que las utiliza para la mejora en el diseño y desarrollo de los MOOC es AbiertaUGR. 


\section{SATISFACCIÓN DE LOS PARTICIPANTES CON LA EVALUACIÓN EN MOOC}

Para dar respuesta al segundo objetivo de la investigación se analiza la opinión de los participantes sobre el sistema de evaluación en el caso de los MOOC de AbiertaUGR.

El cuestionario de valoración cumplimentado tras finalizar los cursos pedía a los participantes la evaluación de su experiencia formativa en conjunto. La satisfacción global, medida en escala de 0 -10, es de $8,8.5$ y 8.3 respectivamente. Se ha analizado la información recogida en la pregunta abierta incluida en el cuestionario para obtener la opinión de los participantes sobre la evaluación realizada y aspectos para su mejora. Refleja su importancia en el diseño pedagógico que, del total de 553 unidades temáticas analizadas en los tres cursos, 125 están referidas a la evaluación (22.6\%). La distribución del contenido de las aportaciones aparece en la Figura 7 de acuerdo a las categorías.

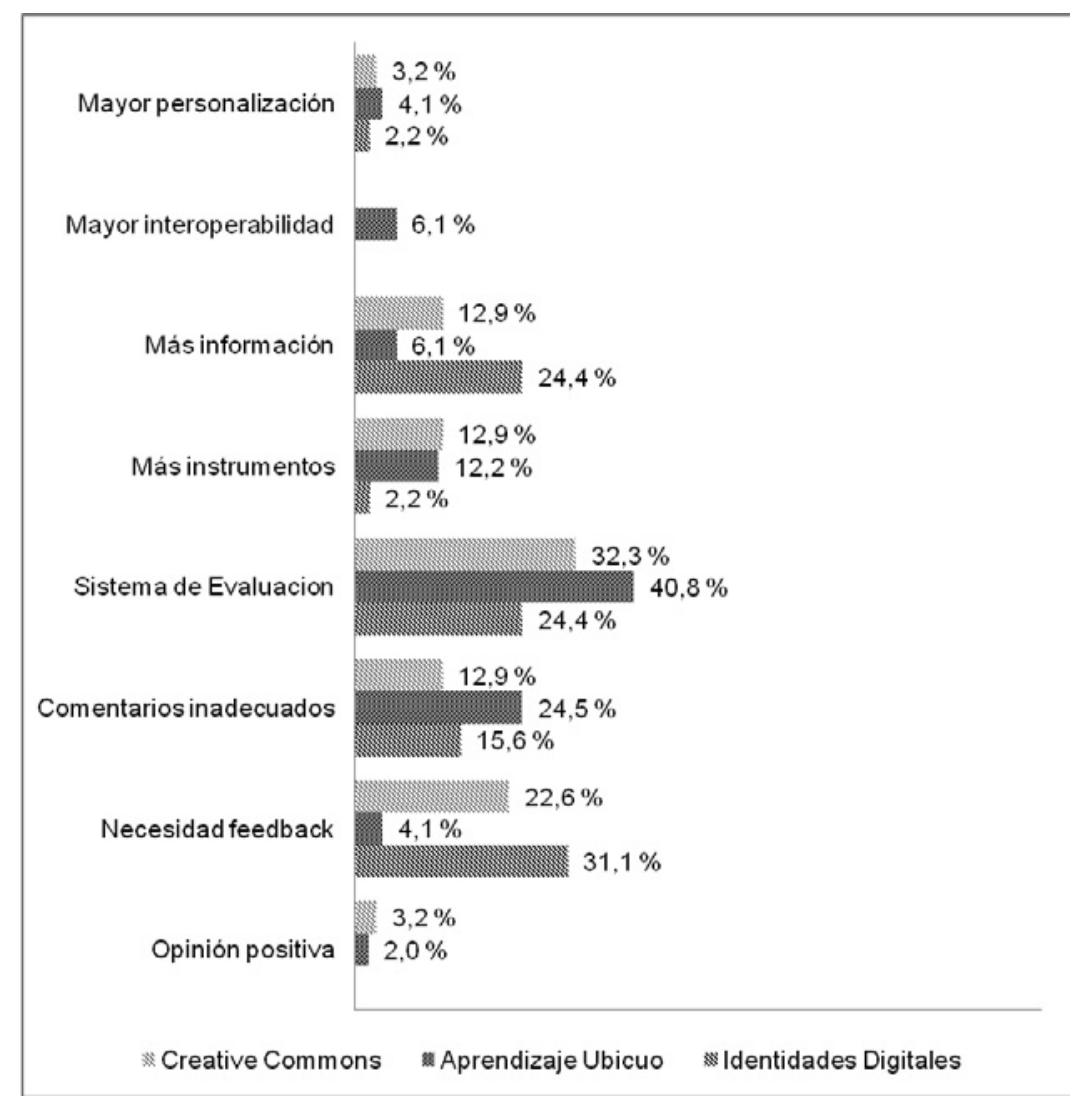

Figura 7. Elementos de mejora en la evaluación en los MOOC de AbiertaUGR 
En los cursos sobre aprendizaje ubicuo $(40,8 \%)$ y creative commons $(32,3 \%)$ aparece el sistema de evaluación con mayor frecuencia, mientras que en el tercero parece ser mayor la necesidad de feedback $(31,1 \%)$. En conjunto una de cada tres aportaciones en los tres cursos hace referencia al sistema de evaluación que consideran, en general, mejorable.

Aparecen comentarios como «Me ha parecido muy limitado que se concedan puntos solo por los comentarios» [UT_27] o "... son necesarios demasiados puntos para obtener «el apto» [UT_41].

El sistema de evaluación provoca comentarios inadecuados en el foro y escasa atención a la calidad de las aportaciones. "... he visto que mucha gente repetía comentarios o comentaba cosas triviales solo por conseguir más puntos.»[UT_11] o "...este sistema de valoración afecta también a la calidad de los comentarios... ya que hay que publicar un número excesivo de ellos, muchos son gratuitos e innecesarios...»[UT_169].

Entre las soluciones que plantean, la de mayor frecuencia es que se produzca un aumento del feedback que incida en el proceso de aprendizaje y que les aporte mayor interactividad y comunicación con los dinamizadores. También mejorarían la experiencia con mayor información sobre la evaluación (objetivos, criterios...) y aumentando los instrumentos de evaluación con cuestionarios semanales o evaluación por pares, «algún tipo de autoevaluación para los contenidos más importantes, evaluación P2P con rúbricas, etc...»[UT_192]. También solicitan mayor adaptación de la evaluación a cada uno de los participantes y mayor interoperabilidad entre plataformas y cursos.

\section{DISCUSIÓN Y CONCLUSIONES}

Este estudio exploratorio analiza la evaluación del aprendizaje en 87 MOOC y la satisfacción ante la evaluación de los participantes en cursos de AbiertaUGR. La evaluación del aprendizaje de los participantes merece una atención especial debido a su impacto en la construcción del conocimiento. El objetivo es analizar el qué, quién, cuándo y cómo de la evaluación del aprendizaje en los cursos.

En las plataformas analizadas se observa un diseño pedagógico poco colaborativo, centrado en conocimientos y contenidos, frente a un porcentaje bajo centrado en la participación o el aprendizaje colaborativo (Aguaded, Vázquez-Cano \& Sevillano-García, 2013). 
Se hace necesario el establecimiento de otros sistemas que sustituyan el tradicional rol evaluador desarrollado por el profesorado. En esta investigación se han encontrado en un porcentaje muy alto cursos que evalúan a través de sistemas automáticos y evaluación por pares (Sandeen, 2013; Balfour, 2013), sistema evaluador que, según Glance, Forsey \& Riley (2013), no resta valor, fiabilidad ni precisión a los resultados ya que se aproximan bastante a la evaluación realizada por un profesor. En relación con el momento evaluativo un $50 \%$ aproximadamente de los cursos analizados realizan varias evaluaciones a lo largo del curso o bien son organizadas semanal o quincenalmente o según el diseño instruccional (por módulos o temas) frente a un 35\% con solo una al finalizar el curso. Existe gran variedad de herramientas para la evaluación que se acomodan a la temática de cada curso, a las preferencias de los diseñadores y a las posibilidades que ofrece cada plataforma de MOOC. Las herramientas más utilizadas son actividades, cuestionarios, exámenes y tareas, frente a accesos y medallas sociales, que son menos frecuentes. Es escasa la diversidad de herramientas; más del 50\% de los MOOC analizados utilizan únicamente una herramienta. Los tipos de evaluación hallados son esencialmente de carácter normativo y continuo.

La importancia de la evaluación no solo preocupa a promotores, instituciones y diseñadores de los MOOC. Según nuestros resultados en el 16.13\% de los MOOC se evalúa la satisfacción del participante, pero también preocupa aproximadamente a uno de cada tres el sistema evaluador, así como los comentarios inadecuados, la necesidad de feedback y contar con más instrumentos (Figura 7) (Sun, Tsai et al., 2008; Mackness, Mak \& Willians, 2010).

De esta forma, teniendo en cuenta la investigación realizada hasta el momento, el análisis de las evaluaciones en MOOC y la opinión del participante, se considera pertinente plantear una serie de propuestas de mejora de los sistemas evaluativos en este formato de cursos masivos:

- Aumentar la variedad de herramientas de evaluación para una mayor adaptación a diferentes formas de aprendizaje.

- Acomodar el tipo de herramientas a los objetivos de aprendizaje.

- Orientar el sistema evaluativo hacia competencias y aprendizaje colaborativo.

- Implementar sistemas evaluativos que potencien la interactividad y el feedback entre los participantes.

- Potenciar el autoaprendizaje a través de la corrección automática de las pruebas y cuestionarios. 
- Estructurar los foros de discusión para una mayor claridad, facilidad de lectura y de construcción del conocimiento en ellos.

- Escalonar los sistemas de puntaje de acuerdo al trabajo realizado por el participante.

- Establecer sistemas de alerta para evitar y/o anular las participaciones vacías de contenido.

- Potenciar el feedback.

- Mayor interoperatividad.

Se recomienda que las instituciones que están poniendo en funcionamiento cursos online masivos proporcionen mayor información sobre los sistemas de evaluación del curso y los requerimientos para alcanzar los diferentes tipos de certificación, incluyendo con carácter previo información pedagógica junto a la administrativa (pago, plazo, etc). Además, dada la variedad de actitudes, conocimientos y destrezas de los participantes online, se recomienda especialmente diversificar los sistemas de evaluación, orientando en la medida de lo posible la elección del sistema de evaluación y el esfuerzo hacia metas concretas ajustadas a sus perfiles.

Debido al muestreo no aleatorio mediatizado por el procedimiento de recomendación del buscador Google y el juicio de los participantes en la Fase 1, el presente estudio exploratorio presenta la limitación de una muestra representativa pero generalizable, en sentido estricto.

En cualquier caso, el uso de la e-evaluación en MOOC está en la agenda de investigación para el futuro (Sandeen, 2013), junto con el uso de learning analytics o la difuminación de las fronteras entre la enseñanza, la evaluación y el aprendizaje (Jordan, 2013). Se estima la necesidad de un mayor número de estudios sobre e-evaluación en cursos online así como estudios longitudinales (Stödberg, 2012) o comparativos (Balfour, 2013). También estudios que analicen el comportamiento de participantes con distintas características (taxonomía de Standford) con el uso de diferentes tipos de evaluación y tasas de finalización (Haggard, 2013; Jordan, 2014). Y, más concretamente, continuar investigando para dar respuesta a preguntas sobre métodos que mejoren fiabilidad, validez, autenticidad y seguridad de las evaluaciones del estudiante, o sobre técnicas que ofrezcan evaluación automatizada eficaz y sistemas de retroalimentación inmediata; y cómo pueden ser integrados en ambientes de aprendizaje abiertos (Oncu \& Cakir, 2011). 


\section{REFERENCIAS BIBLIOGRÁFICAS}

Agarwala, M. (2013). A research summary of MOOC completion rates. EdLab: Teachers College (Columbia University). Recuperado de edlab.tc.columbia.edu/index. php?q=node $/ 8990$

Aguaded, J. I., Vázquez-Cano, E. y Sevillano-García, M. L. (2013). MOOCs, ¿turbocapitalismo de redes o altruismo educativo? Hacia un modelo más sostenible. SCOPEO INFORME, 2. MOOC: Estado de la situación actual, posibilidades, retos y futuro, 74-90. Recuperado de scopeo.usal.es/wp-content/uploads/2013/06/scopeoi002.pdf

Al-Smadi, M. \& Guetl, C. (2011). Supporting self-regulated learners with formative assessments using automatically created QTI-questions. Paper presented at the IEEE Global Engineering Education Conference, EDUCON 2011, 288-294. doi: 10.1109/EDUCON.2011.5773150

Anderson, T. \& McGreal, R. (2012). Disruptive Pedagogies and Technologies in Universities. Educational Technology \& Society, 15(4), 380-389.

Baggaley, J. (2013). MOOC rampant. Distance Education, 34(3), 368-378. doi: 10.1080/01587919.2013.835768

Balfour, S. P. (2013). Assessing writing in MOOCs: Automated essay scoring and Calibrated Peer Review. Research \& Practice in Assessment, 8(1), 40-48.

Brink, R. \& Lautenbach, G. (2011). Electronic assessment in higher education. Educational Studies, 37(5), 503-512. doi: 10.1080/03055698.2010.539733

Cebrián, M. (Coord.). (2012). E-rúbrica federada para la evaluación de los aprendizajes. In C. Leite \& M. Zabalza (Eds.). Ensino Superior. Inovaçao e qualidade na docencia (pp.405-486). Porto: Universidade do Porto.

Cheng, A. C., Jordan, M. E., \& Schallert, D. L. (2013). Reconsidering assessment in online/hybrid courses: Knowing versus learning. Computers \& Education, 68, 51-59. doi: 10.1016/j. compedu.2013.04.022

Clarà, M., \& Barberà, E. (2013). Learning online: Massive open online courses (MOOCs), connectivism, and cultural psychology. Distance Education, 34(1), 129-136. doi: 10.1080/01587919.2013.770428

De la Herrán, A. (2014). La evaluación didáctica. En F. CARRATALÁ (Dir.). Evaluar para mejorar, (13-28). Madrid: Boletín del COFLC.

Earl, K. (2013). Student views on short-text assignment formats in fully online courses. Distance Education, 34(2), 161-174. doi: 10.1080/01587919.2013.793639

Gallego-Arrufat, M. J. \& GutiérrezSantiuste, E. (2015). Perception of democracy in computer-mediated communication: participation, responsibility, collaboration, and reflection. Teaching in Higher Education, 20(1), 92-106. doi: 10.1080/13562517. 2014.957270

Gea, M. \& Montes, R. (2013). AbiertaUGR, la formación abierta basada en comunidades de aprendizaje online. SCOPEO INFORME, 2. MOOC: Estado de la situación actual, posibilidades, retos y futuro, 122-138. Recuperado de scopeo.usal.es/wp-content/ uploads/2013/06/scopeoi002.pdf

Glance, D. G., Forsey, M. \& Riley, M. (2013). The pedagogical foundations of massive open online courses. 
First Monday, 18(5-6). doi: 10.5210/ fm.v18i5.4350

Haggard, S. \& Al. (2013). The maturing of the MOOC: Literature review of massive open online courses and other forms of online distance learning. BIS Research Paper No. 130. Recuperado de www.gov.uk/government/uploads/ system/uploads/attachment_data/ file/240193/13-1173-maturing-of-themooc.pdf

Hoang, L. P. \& Arch-Int, N. (2013). Assessment of Open-Ended Questions using a Multidimensional Approach for the Interaction and Collaboration of Learners in E-Learning Environments. Journal of Universal Computer Science, 19(7), 932-949. doi: 10.3217/ jucs-019-07-0932

Jordan, K. (2014). How many students complete courses? Recuperado de www.katyjordan.com/MOOCproject. html

Jordan, S. (2013). E-assessment: Past, present and future. New Directions, 9(1), 87-106. doi: 10.11120/ ndir.2013.00009

Kerrison, M. (2013). 91\% MOOC satisfaction rating for University of London international programmes. In Massive Open Online Course (MOOC) Report 2013. M2 Communications. Presswire. Recuperado de www.londoninternational.ac.uk/91-Percent-MOOC-satisfaction-rating

King, C., Robinson, A. \& Vickers, J. (2014). Targeted MOOC captivates students. Nature, 505, 7481, 26. doi: $10.1038 / 505026 \mathrm{a}$

Lafuente, M., Remesal, A. \& Álvarez, I. (2014). Assisting Learning in e-Assessment: A Closer Look at Educational Supports. Assessment and Evaluation in Higher Education, 39(4), 443-460. doi: 10.1080/02602938.2013.848835
Lawton, D., Vye, N. \& Al. (2012). Online learning based on essential concepts and formative assessment. Journal of Engineering Education, 101(2), 244-287. doi: 10.1002/j.2168-9830.2012.tb00050.x

Lukas, J. F. y Santiago, K. (2004). Evaluación educativa. Madrid: Alianza Editorial.

Mackness, J., Mak, S. \& Williams, R. (2010). The ideals and reality of participating in a MOOC. In L. DirckinckHolmfeld, V. Hodgson \& Al. (Eds.). Proceedings of the 7th International Conference on Networked Learning (pp.266275). Lancaster: University of Lancaster.

McDaniel, R., Lindgren, R. \& Friskics, J. (2012). Using badges for shaping interactions in online learning environments. Paper presented at the IEEE International Professional Communication Conference. doi: 10.1109/ IPCC.2012.6408619

Meyer, J. P. \& Zhu, S. (2013). Fair and equitable measurement of student learning in MOOCs: An introduction to item response theory, scale linking, and score equating. Research \& Practice in Assessment, 8(1), 26-39.

Oliver, M., Hernández-Leo, D. y Al. (2014). MOOCs en España. Panorama actual de los cursos masivos abiertos en línea en las universidades españolas. Cátedra Telefónica/Universitat Pompeu Fabra. Recuperado de www.catedratelefonica.upf.edu/wpcontent/uploads/2014/02/MOOCs-enEspa\%C3\%B1a1.pdf

Oncu, S. \& Cakir, H. (2011). Research in online learning environments: Priorities and methodologies. Computers \& Education, 57 (1), 1098-1108. doi: 10.1016/j.compedu.2010.12.009

Ramsaran-Fowdar, R. R., Baguant, P. \& Fowdar, S. (2011). A critical analysis of e-assessment with particular emphasis 
on the use of different types of online quizzes. International Journal of Learning, 18(1), 191-202.

Sandeen, C. (2013). Assessment's place in the new MOOC world. Research \& Practice in Assessment, 8(1), 5-12.

Saul, C. \& Wuttke, H. D. (2012). Towards a high-level integration of interactive tools with e-assessments. Paper presented at the IEEE 12th International Conference on Advanced Learning Technologies (ICALT, 2012) (pp.597-598). Rome (Italy). doi: 10.1109/ICALT.2012.169

SCOPEO (2013). INFORME N. ${ }^{\circ} 2$. MOOC: Estado de la situación actual, posibilidades, retos y futuro. Recuperado de scopeo.usal.es/wp-content/ uploads/2013/06/scopeoi002.pdf

Shank, P. (2012). Four Typical Online Learning Assessment Mistakes. In R. Kelly (Ed.) Online Classroom. Report Assessing online learning: Strategies, challenges, opportunities (pp.4-6). Magna Publications. Recuperado de www.facultyfocus.com/wp-content/uploads/images/AssessingOnlineLearning-OC.pdf

Siemens, G. (2012). MOOCs are really a platform. [Web log message]. Recuperado de www.elearnspace.org/ blog/2012/07/25/moocs-are-really-aplatform/
Sorensen, E. (2013). Implementation and student perceptions of e-assessment in a chemical engineering module. European Journal of Engineering Education, 38(2), 172-185. doi: 10.1080/03043797.2012.760533

Stödberg, U. (2012). A research review of e-assessment. Assessment and Evaluation in Higher Education, 37(5), 591-604. doi: 10.1080/ 02602938. 2011.557496

Sun, P. C. et al. (2008). What drives a successful e-Learning? An empirical investigation of the critical factors influencing learner satisfaction. Computers \& Education, 50(4), 1183-1202. doi: 10.1016/j.compedu.2006.11.007

Vonderwell, S. K. \& Boboc, M. (2013). Promoting Formative Assessment in Online Teaching and Learning. TechTrends, 57(4), 22-27. doi: 10.1007/ s11528-013-0673-x

Yuste, R., Alonso, L. \& Blázquez, F. (2012). La e-evaluación de aprendizajes en educación superior a través de aulas virtuales síncronas. Comunicar, 39, 159-167. doi: 10.3916/C39-2012-03-06

Zutshi, S., O’Hare, S. \& Rodafinos, A. (2013). Experiences in MOOCs: The perspective of students. American Journal of Distance Education, 27(4), 218227. doi: 10.1080/08923647.2013.838067 


\section{PERFIL ACADÉMICO Y PROFESIONAL DE LAS AUTORAS}

María Jesús Gallego Arrufat, Doctora en Filosofía y Ciencias de la Educación. Profesora en la Universidad de Granada. Facultad de Ciencias de la Educación. Departamento de Didáctica y Organización Escolar.

Vanesa Gámiz Sánchez, Doctora en Pedagogía. Profesora en la Universidad de Granada. Facultad de Ciencias de la Educación. Departamento de Didáctica y Organización Escolar.

Elba Gutiérrez Santiuste, Doctora en Ciencias de la Educación. Profesora en la Universidad de Córdoba. Facultad de Educación. Departamento de Educación.

Dirección de las autoras: María Jesús Gallego Arrufat Vanesa Gámiz Sánchez

Facultad de Ciencias de la Educación

Universidad de Granada

Campus de Cartuja, s/n

18071 Granada, España

E-mail: mgallego@ugr.es vanesa@ugr.es

Elba Gutiérrez Santiuste

Facultad de Educación

Universidad de Córdoba

San Alberto Magno, s/n

14071 Córdoba, España

E-mail: egsantiuste@uco

Fecha Recepción del Artículo: 12. Noviembre. 2014

Fecha Modificación Artículo: 25. Enero. 2015

Fecha Aceptación del Artículo: 26. Enero. 2015

Fecha Revisión para publicación: 30. Enero. 2015 